In two lectures Prof. Wesley Cocker, University professor of chemistry, Trinity College, Dublin, dealt with new reagents and techniques. He criticized the old idea that the aldehyde-Girard products are more stable than the ketonic adducts and went on to describe newer methods for isolating carbonyl compounds. He then described modern specific catalysts for reducing alkene and carbonyl compounds. Recent oxidizing agents which are specific for various groups were outlined, and in conclusion the work of Linstead and others who have produced long-chain fatty acids by a variation of the Kobe electrolytic synthesis of paraffins was described.

In the two hours allotted to him, Mr. P. W. D. Mitchell, Lasdon Fellow of the Medical Research Council of Ireland, described briefly the symbols and terminology used in absorption. spectroscopy. $\mathrm{H}_{\theta}$ indicated how the positions of the regions of absorption and the intensity might be correlated empirically with the nature and distribution of chromophores and auxochromes in the molecule and then described some work in which he is engaged for the Medical Research Council of Ireland. In this work the ultraviolet absorption spectra are used as a means of determining molecular weights and also as a method for the identification of sugar osazones.

Dr. E. F. McCarthy, senior Fellow of the Medical Research Council of Ireland, outlined recent advances in electrophoretic technique and its application to the separation of high- and low-molecular-weight substances of biological importance. He discussed the relative merits of boundary and zone electrophoresis with reference to his own work on the identification of blood proteins. Dr. McCarthy also described the more recent refinements in the estimation of molecular weights by osmotic pressure measurements.

Two lectures on "Distillation" were delivered by Mr. F. T. Riley, lecturer in chemistry in University College, Dublin, dealing with liquid-vapour equilibria and with the theoretical basis of modern laboratory. fractional distillation. Laboratory fractionation practice in the 1920's was contrasted with presentday methods. Mr. Riley emphasized the changes brought about by the introduction of controlled reflux, and packed columns. He then reviewed the factors influencing separation efficiency and described the high-efficiency column working in the Department of Chemistry of University College, Dublin. This column has a packed length of $9 \mathrm{ft}$., and its performance is equivalent to that of a column containing at least two hundred theoretical plates. A feature of this column is the intermittent vapour draw-off device which is useful with an azeotropic mixture that separates on condensation.

Prof. F. J. Coll, professor of chemistry in University College, Galway, gave two lectures on "Chromatography" and discussed this technique under the following headings: absorption chromatography, partition chromatography, paper chromatography, ion-exchange chromatography and electrochromatography. Many excellent paper chromatograms of sugars, prepared by Prof. Coll, were used for illustrating the points described. Other experimental demonstrations were much admired.

Two lectures on organic chemistry and analysis were given by Dr. Cecil L. Wilson, reader in analytical chemistry in The Queen's University, Belfast. Beginning with a review of the development of analytical chemistry, he went on to discuss the use of micromethods in the laboratory. He described the appli- cation of organic reagents in gravimetric, titrimetric and colorimetric procedures and showed how the requirements for a 'classical' organic reagent have been modified by the addition to this class of the 'complexones', which cannot be regarded as following the accepted pattern. The inorganic analyst, in particular, is bound, in the future, to take more note of the methods of his organic colleague, since he will more and more be faced with problems of compound analysis rather than of ion analysis.

Mr. C. D. O'Briain, Department of Chemistry, University College, Dublin, organized an excellent display of scientific apparatus, the material being supplied by a number of manufacturers and agents. Eva M. Philbin

\section{PHYSICS IN ISRAEL}

THE seventy-fifth birthday of Prof. Albert Einstein was celebrated in Israel by a scientific convocation which was held on March 14 in Jerusalem, under the joint auspices of the Hebrew University of Jerusalem; the Technion, Haifa; the Weizmann Institute of Science, Rehovot; and the Research Council of Israel. Tributes were paid to Prof. Einstein by Mr. Y. Ben-Zvi, the Israeli president, Mr. M. Sharett, the Israeli prime minister, and Prof. B. Mazur, president of the Hebrew University, and various aspects of Prof. Einstein's contributions to science were discussed by Prof. G. Racah of the Hebrew University (quantum theory), Prof. C. Pekeris of the Weizmann Institute (Brownian movement), Prof. $N_{k}$ Rosen of the Technion (relativity) and Prof. S. Sambursky, director of the Research Council (cosmology).

In addition, the March number of the Bulletin of the Research Council of Israel (3, No. 4; 1954) is dedicated "with the highest esteem and affection of the scientists of Israel" to Einstein in honour of his birthday, and contains a photograph of Prof. Einstein as frontispiece. The contents consist of twenty-four original contributions, mainly devoted to mathematics or theoretical physies, the abstracts of the papers presented at the inaugural meeting of the Israel Physical Society held during April 12-13, 1954, and six short research notes. The Bulletin, which is in English, contains, in addition, abstracts of the original contributions in both Hebrew and English.

The inaugural meeting of the Physical Society consisted of a general session, opened by the Society's first president, Prof. G. Racah, at which two addresses were delivered, one by E. D. Bergmann on the influence of modern physics on organic chemistry and the other by M. Chwalow on the role of physics in industry, and four other sessions devoted to general and industrial physics; theoretical physics ; atomic, nuclear and molecular physics; and methods and instruments. The abstracts indicate that work in the experimental field of physies is gradually being built up alongside the numerous theoretical studies, and show that recent activities include the construction of a thin lens magnetic $\beta$-ray spectrometer, and of a Couette viscometer for precision measurement in the centipoise ranges, the measurement of the absorption and scattering of $1-3 \mathrm{~cm}$. radiation by a d.c. discharge in helium and air using a low-powered klystron as microwave generator, and experiments on luminescence. 